\title{
Major Discordant Structural Anomalies in Monochorionic Twins: Spectrum and Outcomes
}

\author{
Maria Angela Rustico, ${ }^{1}$ Mariano Lanna, ${ }^{1}$ Stefano Faiola, ${ }^{1}$ Daniela Casati, ${ }^{1}$ Luigina Spaccini, ${ }^{2}$ \\ Andrea Righini, ${ }^{3}$ Cecilia Parazzini, ${ }^{3}$ Marcello Napolitano, ${ }^{3}$ Barbara Scelsa, ${ }^{4}$ Gianluca Lista, ${ }^{5}$ Carla Corti, ${ }^{6}$ \\ Giovanna Riccipetitoni, ${ }^{7}$ and Irene Cetin ${ }^{8}$ \\ ${ }^{1}$ Fetal Therapy Unit 'Umberto Nicolini', Vittore Buzzi Children's Hospital, Università di Milano, Milan, Italy \\ ${ }^{2}$ Clinical Genetics Service, Vittore Buzzi Children's Hospital, Università di Milano, Milan, Italy \\ ${ }^{3}$ Department of Radiology and Neuroradiology, Vittore Buzzi Children's Hospital, Università di Milano, Milan, Italy \\ ${ }^{4}$ Pediatric Neurology Unit, Vittore Buzzi Children's Hospital, Università di Milano, Milan, Italy \\ ${ }^{5}$ Neonatal Intensive Care Unit, Vittore Buzzi Children's Hospital, Università di Milano, Milan, Italy \\ ${ }^{6}$ Pediatric Cardiology Unit, Vittore Buzzi Children's Hospital, Università di Milano, Milan, Italy \\ ${ }^{7}$ Department of Pediatric Surgery, Vittore Buzzi Children's Hospital, Università di Milano, Milan, Italy \\ ${ }^{8}$ Department of Obstetrics and Gynecology, Vittore Buzzi Children's Hospital, Università di Milano, Milan, Italy
}

\begin{abstract}
Monochorionic twins, resulting from a single fertilized egg giving rise to two separate embryos, are monozygotic and considered genetically identical. However, discordant phenotypes have been reported in monozygotic twins. We analyzed a retrospective cohort of 155 monochorionic pregnancies (312 twins) with major discordant structural anomalies coded by the ICD-10 system in order to describe the spectrum of anomalies, the management of the pregnancies, and the perinatal outcome. Treatment options included conservative management, selective feticide with bipolar cord coagulation, or complete termination. All survivors underwent at least 24 months of postnatal follow-up. Discordancy was complicated by twin-to-twin transfusion syndrome in eight pregnancies (5\%) and by selective intrauterine growth restriction in 41 (26\%). Major structural anomalies affected one system in 139 cases (90\%) and multiple systems in 16 (10\%). Median gestational age at diagnosis was 19.1 weeks (IQR 16.4-21.3). The most frequent single-system anomalies involved the nervous and circulatory systems. In total, 72 anomalous twins (46\%) and 116 normal co-twins (74\%) were delivered at a median gestational age of 34.6 weeks (IQR 31.0-36.3). Neonatal/infant death of the anomalous twin occurred in 22 cases (14\%), with an overall survival rate of $32 \%(50 / 155)$. Surviving anomalous twins underwent major surgery in $22 / 50$ cases (44\%), four of whom ( $8 \%$ ) now suffer from severe neurologic morbidity. This study shows that a wide spectrum of major discordant structural anomalies can be found in monochorionic pregnancies. The outcome for the anomalous twin is poor, while the survival rate for the normal co-twin was $71 \%$, with a favorable overall prognosis.
\end{abstract}

Keywords: monochorionic twins, discordant structural anomalies, management, perinatal outcome

Data from population-based surveys have confirmed previous observations reporting an excess of congenital anomalies - nearly twice as many - in monochorionic (MC) compared to dichorionic (DC) twins and to singletons (5$6 \%$ in the former, compared to $2-3 \%$ in the latter; Glinianaia et al., 2008; Hall, 2003). Together with the more common complications of MC pregnancies, namely twinto-twin transfusion syndrome (TTTS) and selective intrauterine growth restriction (sIUGR), congenital anomalies also contribute to the increased rate of perinatal morbidity and mortality observed in MC pregnancies (Alexander et al., 1997; Pharoah \& Dundar, 2009; Wood et al., 2014). MC twins, resulting from a single fertilized egg giving rise to two separate embryos, are monozygotic (MZ) and consid- ered genetically identical. For this reason, when congenital anomalies are present they are expected to affect both twins. However, many studies have reported that MZ twins are not genetically identical, and may be discordant for chromosomal anomalies, Mendelian disorders, and other important phenotypes, including structural anomalies (Machin,

RECEIVED 13 June 2018; ACCEPTEd 20 September 2018. First published online 30 October 2018.

ADDRESS FOR CORRESPONDENCE: Maria Angela Rustico, Fetal Therapy Unit 'Umberto Nicolini', Vittore Buzzi Children's Hospital, Università di Milano, via Castelvetro 32-20154 Milano, Italy. E-mail: cast@interware.it 
2009; Silva et al., 2011). These findings have given rise to intense debate about the genetic, epigenetic, and environmental origins of the discordance, a debate that goes beyond the scope of the present study (Castillo-Fernandez et al., 2014; Zwijnenburg et al., 2010). Obstetricians involved in the surveillance of MC twins are well aware that these fetuses are not only at increased risk of congenital structural anomalies, but that these anomalies can also occur discordantly and require a careful and complex clinical management (Gratacos et al., 2012; Khalil et al., 2016).

Although there have been numerous studies describing MC discordancies for abnormal karyotype and for cardiac and non-cardiac malformations (often comprising of minor findings such as single umbilical artery, choroid plexus cyst, and pyelectasis), these are limited to single case reports or to small series complicated or not by TTTS (Sperling et al., 2007; Springer et al., 2014). The primary aim of this study was to describe the spectrum of major discordant structural anomalies observed in a large cohort of MC pregnancies, coded using a standardized system to make classification as objective as possible. The secondary objective was to describe the management of these pregnancies and the perinatal outcome of both anomalous and normal MC twins.

\section{Materials and Methods}

\section{Case Selection}

The study complied with our Institution's research guidelines for clinical observational and retrospective studies.

This is a retrospective descriptive analysis of all consecutive MC pregnancies with major discordant structural anomalies referred after 14 weeks' gestation to the 'Umberto Nicolini' Fetal Therapy Unit of the V. Buzzi Children's Hospital, University of Milan, Italy, between January 2004 and December 2015. All reported pregnancies were diagnosed as $\mathrm{MC}$ at first trimester ultrasound. Over the 12-year study period, 1,750 MC pregnancies were referred to our unit, including 485 complicated with TTTS. In total, 168 of these pregnancies were managed for major structural anomalies observed either in both twins (13 pregnancies) or in one (155 pregnancies). This cohort of 155 pregnancies is the object of the present study. All prenatal records were reviewed and the pregnancies were divided as follows: $\mathrm{MC}$ diamniotic (MC DA), MC triamniotic (MC TA), MC monoamniotic (MC MA), and DC triamniotic (DC TA). Pregnancies complicated with twin-reversed arterial perfusion (TRAP) sequence, conjoined twins, or intrauterine fetal death (IUFD) of one or both twins at referral were excluded from the study. Structural anomalies were defined as major when one MC twin presented at ultrasound with a lethal malformation, or when the detected anomaly required postnatal surgery or was associated with the increased risk of functional or neurological impairment. We also recorded when the discordant anomaly occurred in pregnancies complicated with sIUGR (defined as previously described; Rustico et al., 2017), whether the affected twin was the small or the large one, or when it occurred in TTTS pregnancies, and whether the affected twin was the donor or the recipient. In this study, TTTS-related recipient twin cardiomyopathy (cardiomegaly, ventricular hypertrophy, atrio-ventricular valvular regurgitation, and right ventricle outflow tract obstruction; Manning \& Archer, 2016; Rustico et al., 2012) was not considered as a discordant cardiac anomaly. Ultrasound findings such as single umbilical artery, choroid plexus cyst, pyelectasis, or stable borderline ventriculomegaly (less than $12 \mathrm{~mm}$ ) were also not considered.

\section{Case Diagnosis and Clinical Work-Up}

A detailed anatomical ultrasound evaluation, including echocardiography, was carried out of the MC twins, as well as three-dimensional ultrasound examination of the fetal brain starting from the second trimester (GE Voluson 730 Expert, and GE E8, GE Healthcare, Zipf, Austria). We also carried out serial fetal biometry, including Doppler interrogation of the umbilical artery, ductus venosus, and middle cerebral artery. Whenever the major structural anomaly was identified in one twin in conjunction with normal ultrasound findings in the co-twin, fetal karyotyping (by amniotic fluid sampling of both amniotic sacs) was offered if not previously acquired (Gentilin et al., 2008). Until 2010, conventional karyotyping was performed, subsequently replaced by array comparative genomic hybridization analysis (aCGH; Hilmann et al., 2013). In the presence of anomalies affecting the central nervous system (except for cases of anencephaly, encephalocele, holoprosencephaly, and spina bifida), of congenital pulmonary airway malformations (CPAM, formerly congenital cystic adenomatoid malformations or CCAM) and congenital diaphragmatic hernia $(\mathrm{CDH})$, in utero magnetic resonance imaging (MRI) was also performed alongside ultrasound diagnosis (Conte et al., 2016). All families received extensive counseling regarding the management options for discordant anomalies: selective feticide with bipolar cord coagulation (BCC; Lanna et al., 2012), complete termination of the pregnancy (TOP), or conservative management. Issues addressed included the risk of co-twin loss and the increased risk of premature delivery after BCC, along with the option of conservative management. Patients with potentially correctable anomalies complicated with both TTTS were told about the option of fetoscopic laser surgery (FLS; Slaghekke et al., 2014). Autoptic examination was performed in all cases that ended in miscarriage or TOP, except for four cases of early termination.

All surviving infants, with and without prenatally detected major structural anomalies, were followed up with serial neurological examinations by a pediatric neurologistpsychiatrist, which is routine practice in Italy, for at least 24 months, with the exception of two cases lost some months after cardiac surgery (Marlow, 2013). Neonates/infants without standard karyotype/aCGH but with normal clinical assessment were considered to be normal. 


\section{Data Analysis}

To produce a standardized classification of our cases and make the description as objective as possible, each anomaly was coded and named retrospectively using the International Statistical Classification System of Diseases and Related Health Problems, tenth revision (ICD-10; World Health Organization [WHO], 2016). The classification of structural congenital anomalies is reported in Chapter XVII (Congenital Malformations, Deformations and Chromosomal Abnormalities), and contains a range of blocks of codes from Q00 to Q99.

Those anomalies familiar to prenatal ultrasonographers but not envisaged by the ICD-10 classification system, such as severe ventriculomegaly not due to obstruction of the ventricular system (for which there is a specific code), or vermian hypoplasia not classifiable as Dandy-Walker syndrome (which also has its own code), are shown in our tables in italics. Other anomalies not included (such as sacrococcigeal teratoma or romboencephalosynapsis) have been inserted in the blocks entitled 'congenital malformation, and unspecified'. Anomalies affecting one system were reported separately from those affecting multiple systems. When multiple congenital anomalies were present, a detailed description of each major malformation was recorded, as suggested in the ICD-10 Manual for the Surveillance of Congenital Anomalies.

\section{Results}

Table 1 shows the antenatal characteristics at diagnosis of the 155 pregnancies ( 312 MC twins) with major discordant structural anomalies included in the study (139 affecting one system and 16 affecting multiple systems), followed by perinatal outcome. Median gestational age (GA) at diagnosis was 19.1 weeks (IQR 16.4-21.3), with $88 \%$ of cases identified before 22 weeks' gestation. Karyotyping was performed in $94(61 \%)$ cases. In the other cases, the women chose not to perform amniocentesis either because of advanced gestational age at diagnosis, out of personal choice, or to avoid the possible risks of complications for the co-twin.

Major discordant structural anomalies were associated in $41(26 \%)$ cases with sIUGR, and in $8(5 \%)$ cases with TTTS. IUFD of both twins occurred in 11 pregnancies (7\%). In 5 cases (3\%), single IUFD of the normal twin occurred and five anomalous co-twins were delivered. Overall, 55 (35\%) pregnancies were managed by BCC of the anomalous twin. Co-twin loss subsequent to this procedure occurred in eight cases (5\%). One pregnancy miscarried, while $16(10 \%)$ were terminated. A total of 72 anomalous twins and 116 normal co-twins (two MC TA pregnancies had two normal co-twins) were delivered at a median GA of 34.6 weeks (IQR 31.0-36.3). A total of 22 twins (14\%) with structural anomalies died in the neonatal/infant pe-

\section{TABLE 1}

Antenatal Characteristics at Diagnosis and Perinatal Outcome in 155 MC Pregnancies (312 MC Twins) Complicated with Major Discordant Structural Anomaly

\begin{tabular}{|c|c|}
\hline \multicolumn{2}{|l|}{ Characteristics } \\
\hline Maternal age, years (IQR) & $32(29-36)$ \\
\hline Nulliparous $n(\%)$ & $91(59)$ \\
\hline IVF pregnancies $n(\%)$ & $10(6)$ \\
\hline \multicolumn{2}{|l|}{ Type of MC pregnancies $n(\%)$} \\
\hline MC diamniotic & $127(82)$ \\
\hline $\mathrm{MC}$ monoamniotic & $19(12)$ \\
\hline MC triamniotic & $2(1)$ \\
\hline DC triamniotic & 7 (5) \\
\hline Invasive prenatal diagnosis n (\%) & $94(61)$ \\
\hline GA at diagnosis, weeks & $19.1(16.4-21.3)$ \\
\hline Structural anomaly affecting one system $n(\%)$ & $139(90)$ \\
\hline $\begin{array}{l}\text { Structural anomaly affecting multiple systems } \\
n(\%)\end{array}$ & $16(10)$ \\
\hline Diagnosis $<22$ weeks $n(\%)$ & $137(88)$ \\
\hline Male sex $n(\%)$ & $62(40)$ \\
\hline $\begin{array}{l}\text { Anomalies in pregnancy complicated with } \\
\text { slUGR } n(\%)\end{array}$ & $41(26)$ \\
\hline Affecting small twin $n(\%)$ & $36(23)$ \\
\hline Affecting large twin $n(\%)$ & $5(3)$ \\
\hline $\begin{array}{l}\text { Anomalies in pregnancy complicated with } \\
\text { TTTS } n(\%)\end{array}$ & $8(5)$ \\
\hline Affecting recipient twin $n(\%)$ & $4(2.5)$ \\
\hline Affecting donor twin $n(\%)$ & $4(2.5)$ \\
\hline IUFD of both twins (\%) & $11(7)$ \\
\hline IUFD of normal co-twin $n$ (\%) & $5(3)$ \\
\hline $\mathrm{BCC}$ of anomalous twin $n(\%)$ & $55(35)$ \\
\hline Co-twin loss after BCC $n(\%)$ & $8(5)$ \\
\hline TOP n (\%) & $16(10)$ \\
\hline Miscarriage $n(\%)$ & $1(0.6)$ \\
\hline GA at delivery, weeks ( $n 119$ pregnancies $^{\mathrm{a}}$ ) & $34.6(31.0-36.3)$ \\
\hline \multicolumn{2}{|l|}{ Birthweight, $g$} \\
\hline Normal co-twin & $2095(1,550-2,676)$ \\
\hline Anomalous twin & $1560(1,090-2,030)$ \\
\hline \multicolumn{2}{|l|}{ Live births } \\
\hline Normal co-twin $n(\%)^{b}$ & 116/157 (74) \\
\hline Anomalous twin $n(\%)$ & $72 / 155(46)$ \\
\hline \multicolumn{2}{|l|}{ Neonatal/infant deaths } \\
\hline Normal co-twin $n(\%)$ & $5 / 157(3)$ \\
\hline Anomalous twin $n(\%)$ & $22 / 155(14)$ \\
\hline \multicolumn{2}{|l|}{ Overall survival } \\
\hline Normal co-twin $n(\%)$ & $111 / 157(71)$ \\
\hline Anomalous twin $n(\%)$ & $50 / 155(32)$ \\
\hline \multicolumn{2}{|l|}{$\begin{array}{l}\text { Surviving infants with severe neurologic } \\
\text { morbidity ( } \mathrm{n} / \text { surviving) }\end{array}$} \\
\hline Normal co-twin n/111 (\%) & $0(0)$ \\
\hline Anomalous twin $n / 50(\%)$ & $4(8)$ \\
\hline \multicolumn{2}{|c|}{$\begin{array}{l}\text { Note: } \mathrm{MC}=\text { monochorionic, } \mathrm{DC}=\text { dichorionic, IVF }=\text { in vitro fertilization, } \\
\mathrm{GA}=\text { gestational age, IUFD = intrauterine fetal death, sIUGR = selec- } \\
\text { tive intrauterine growth restriction, } \mathrm{BCC}=\text { bipolar cord coagulation, } \\
\mathrm{TOP}=\text { termination of pregnancy. Values are indicated with number }(n) \\
\text { and percentage in brackets, or with median and interquartile range } \\
\text { (IQR) in brackets. } \\
\text { an } 119 \text { pregnancies with at least one live birth monochorionic twin. } \\
b_{n} \text { two monochorionic triamniotic pregnancies had two normal live } \\
\text { birth co-twins. }\end{array}$} \\
\hline
\end{tabular}

riod, with an overall survival rate of $32 \%(50 / 155)$. Normal co-twin survival rate was $71 \%(111 / 157)$.

Table 2 is a detailed description of the number and type of major discordant structural anomalies affecting one system $(139 / 155,90 \%)$ coded according to the ICD-10 classification. Of these, 30 were lethal or potentially lethal. The anomalies occurring in one of the twins of MC DA (112), MC TA (2), and DC TA (6) pregnancies are listed in Group 1 ; the anomalies occurring in MC MA pregnancies (19) are 


\section{TABLE 2}

Major Discordant Structural Anomalies Affecting One System (N 139) Classified According to the ICD-10

\begin{tabular}{|c|c|c|c|c|c|c|c|c|c|c|}
\hline Code & Prenatal findings & Total & $\begin{array}{l}\text { Group } \\
1 N(\%)\end{array}$ & $\begin{array}{l}\text { Group } \\
2 N(\%)\end{array}$ & $\begin{array}{l}\text { Associated } \\
\text { findings }\end{array}$ & $\begin{array}{l}\text { Group } 1 \\
\text { pregnancy } \\
\text { outcome }\end{array}$ & $\begin{array}{l}\text { Group } 2 \\
\text { pregnancy } \\
\text { outcome }\end{array}$ & $\begin{array}{l}\text { Group } \\
1 \text { live } \\
\text { births } \\
\mathrm{N}(\%)\end{array}$ & $\begin{array}{l}\text { Group } \\
2 \text { live } \\
\text { births } \\
N(\%)\end{array}$ & Postnatal outcome \\
\hline \multicolumn{11}{|c|}{ Malformations of the nervous system (Q00-Q07) } \\
\hline Q00 & Anencephaly & 9 & 8 & 1 & - & $\begin{array}{l}1 \text { TOP } \\
3 \text { BCC } \\
1 \text { IUFD }\end{array}$ & $1 \mathrm{TOP}$ & 3 & 0 & 3 NND (1 co-twin NND) \\
\hline 001.2 & Occipital encephalocele & 1 & & 1 & - & & & & 1 & Surgery, death at 2 years \\
\hline Q03.0 & $\begin{array}{l}\text { Congenital hydrocephalus } \\
\text { Severe bilateral ventriculomegaly }(1+\text { cortical } \\
\text { anomalies) }\end{array}$ & $\begin{array}{l}10 \\
5\end{array}$ & $\begin{array}{l}9 \\
5\end{array}$ & 1 & $\begin{array}{l}3 \text { small twins } \\
2 \text { small twins }\end{array}$ & $\begin{array}{l}5 \text { BCC } \\
1 \text { IUFD } \\
1 \text { TOP } \\
2 \text { BCC }\end{array}$ & $1 \mathrm{BCC}$ & $\begin{array}{l}3 \\
2\end{array}$ & 0 & $\begin{array}{l}2 \text { NND; } 1 \text { surgery + VSD, follow-up } \\
\text { 1NND (+ cortical anomalies); ( } 1 \text { co-twin NND); } 1 \\
\text { speech impairment, special needs support in } \\
\text { school }\end{array}$ \\
\hline Q03.1 & $\begin{array}{l}\text { Dandy Walker syndrome } \\
\text { Vermian hypoplasia }\end{array}$ & $\begin{array}{l}5 \\
8\end{array}$ & $\begin{array}{l}5 \\
8\end{array}$ & & $\begin{array}{l}2 \text { small twins } \\
7 \text { small twins }\end{array}$ & $4 \mathrm{BCC}$ & & $\begin{array}{l}1 \\
8\end{array}$ & & $\begin{array}{l}1 \text { NND } \\
5 \text { follow-up (1+ASD); } 3 \text { special needs support in } \\
\text { school }\end{array}$ \\
\hline Q04.0 & $\begin{array}{l}\text { Agenesis of corpus callosum } \\
\text { ( } 3 \text { partial agenesis of } C C \text { ) }\end{array}$ & 5 & 3 & 2 & 1 donor & $2 \mathrm{BCC}$ & 2 TOP & 1 & 0 & Follow-up \\
\hline 004.2 & Holoprosencephaly & 1 & 1 & & & $1 \mathrm{BCC}$ & & 0 & & \\
\hline Q04.6 & $\begin{array}{l}\text { Congenital cerebral cyst } \\
\text { Arachnoid cyst }\end{array}$ & 1 & 1 & & & & & 1 & & Follow-up \\
\hline Q04.9 & $\begin{array}{l}\text { Congenital malformation of brain unspecified } \\
\text { Sinus dural malformation } \\
\text { Romboencephalosynapsis }\end{array}$ & 2 & $\begin{array}{l}1 \\
1 \\
1\end{array}$ & & & $\begin{array}{l}1 \mathrm{BCC} \\
1 \mathrm{BCC} \\
1 \mathrm{BCC}\end{array}$ & & $\begin{array}{l}0 \\
0\end{array}$ & & \\
\hline Q05.2 & Spina bifida & 7 & 5 & 2 & $\begin{array}{l}1 \text { small twin } \\
1 \text { recipient }\end{array}$ & $\begin{array}{l}1 \text { TOP } \\
3 \text { BCC } \\
1 \text { IUFD }\end{array}$ & $2 \mathrm{BCC}$ & 0 & 0 & (1 co-twin NND) \\
\hline Total & & 54 & $47(87)$ & $7(13)$ & & & & $19(40)$ & $1(14)$ & \\
\hline \multicolumn{11}{|c|}{ Malformations of eye, ear, face, and neck (Q10-Q18) } \\
\hline Q18.3 & Webbing of neck & 3 & 3 & & & $\begin{array}{l}1 \text { TOP } \\
1 \text { IUFD } \\
1 \text { BCC }\end{array}$ & & 0 & & \\
\hline Total & & 3 & $3(100)$ & & & & & $0(0)$ & & \\
\hline \multicolumn{11}{|c|}{ Malformations of the circulatory system (Q20-Q28) } \\
\hline Q20.1 & Double outlet right ventricle & 2 & 1 & 1 & 2 small twins & $1 \mathrm{BCC}$ & 1 TOP & 0 & 0 & \\
\hline $\mathrm{Q} 20.3$ & Transposition of great vessels (complete) & 1 & 1 & & 1 small twin & & & 1 & & Surgery, follow-up \\
\hline Q20.4 & Single ventricle & 4 & 4 & & & $2 \mathrm{BCC}$ & & 2 & & 2 surgery; 1 lost to follow-up after 1 year \\
\hline \multirow[t]{3}{*}{ Q21.0 } & Ventricular septal defect & 3 & & & & & & & & $1+$ imperforate anus, PSARP, surgery for CHD, lost \\
\hline & Malalignment VSD & & 2 & & & & & 2 & & to follow-up after 1 year; $1+$ severe \\
\hline & Membranous VSD & & 1 & & & & & 1 & & $\begin{array}{l}\text { hypospadias, surgery for CHD and hypospadias } \\
\text { repair } \\
1 \text { follow-up }\end{array}$ \\
\hline \multirow[t]{2}{*}{ Q22.0 } & Pulmonary valve atresia (intact ventricular septum) & 5 & 5 & & 1 large twin & $\begin{array}{l}1 \text { TOP } \\
2 \text { BCC }\end{array}$ & & 2 & & $\begin{array}{l}1 \text { surgery, severe psychomotor delay; } 1 \text { surgery, } \\
\text { death at } 2 \text { months }\end{array}$ \\
\hline & Pulmonary valve atresia (+VSD) & 1 & 1 & & 1 recipient & $1 \mathrm{BCC}$ & & 0 & & \\
\hline
\end{tabular}


TABLE 2

Continued

\begin{tabular}{|c|c|c|c|c|c|c|c|c|c|c|}
\hline Code & Prenatal findings & Total & $\begin{array}{l}\text { Group } \\
1 N(\%)\end{array}$ & $\begin{array}{l}\text { Group } \\
2 N(\%)\end{array}$ & $\begin{array}{l}\text { Associated } \\
\text { findings }\end{array}$ & $\begin{array}{l}\text { Group } 1 \\
\text { pregnancy } \\
\text { outcome }\end{array}$ & $\begin{array}{l}\text { Group } 2 \\
\text { pregnancy } \\
\text { outcome }\end{array}$ & $\begin{array}{l}\text { Group } \\
1 \text { live } \\
\text { births } \\
\mathrm{N}(\%)\end{array}$ & $\begin{array}{l}\text { Group } \\
2 \text { live } \\
\text { births } \\
N(\%)\end{array}$ & Postnatal outcome \\
\hline Q22.1 & Pulmonary valve stenosis & 2 & 2 & & 1 large twin & 1 IUFD & & 1 & & $\begin{array}{l}\text { Cardiologic follow-up, spastic bilateral cerebral } \\
\text { palsy, blindness }\end{array}$ \\
\hline Q22.4 & Tricuspid atresia & 2 & 1 & 1 & 1 small twin & & & 1 & 1 & 1 surgery, follow-up; 1 NND \\
\hline Q22.5 & Ebstein's anomaly of tricuspid valve & 1 & 1 & & 1 recipient & 1 IUFD & & 0 & & \\
\hline Q22.8 & $\begin{array}{l}\text { Other congenital malformations of tricuspid valve } \\
\text { Tricuspid valve dysplasia }\end{array}$ & 3 & 3 & & $\begin{array}{l}1 \text { donor } \\
1 \text { large twin }\end{array}$ & $1 \mathrm{FLS}$ & & 3 & & 3 follow-ups \\
\hline Q23.4 & Hypoplastic left heart syndrome & 2 & 2 & & & $1 \mathrm{BCC}$ & & 1 & & NND \\
\hline Q24.8 & $\begin{array}{l}\text { Other specified malformation of heart } \\
\text { Malformation of myocardium }\end{array}$ & 2 & 2 & & & $2 \mathrm{BCC}$ & & 0 & & \\
\hline Q26.8 & Scimitar syndrome & 2 & 2 & & 1 small twin & & & 2 & & $\begin{array}{l}1+\text { oesophageal atresia repair, death at } 1 \text { week; } 1 \\
\text { follow-up }\end{array}$ \\
\hline Total & & 30 & $28(93)$ & $2(7)$ & & & & $16(57)$ & $1(50)$ & \\
\hline \multicolumn{11}{|c|}{ Malformations of the respiratory system (Q30-O34) } \\
\hline Q33.0 & $\begin{array}{l}\text { Congenital malformations of lung } \\
\text { CCAM microcystic type (monolateral) }\end{array}$ & 4 & 4 & & 1 large twin & & & 4 & & $\begin{array}{l}3 \text { lobectomy, follow-up; } 1 \text { segmental overinflation, } \\
\text { follow-up }\end{array}$ \\
\hline Total & & 4 & $4(100)$ & & & & & $4(100)$ & & \\
\hline \multicolumn{11}{|c|}{ Other congenital malformations of the digestive system (Q38-Q45) } \\
\hline Q39.1 & Esophageal atresia with trachea-esophageal fistula & 1 & 1 & & & & & 1 & & Oesophageal atresia repair, follow-up \\
\hline Q41.0 & $\begin{array}{l}\text { Congenital atresia/stenosis of duodenum Duodenal } \\
\text { obstruction }\end{array}$ & 1 & 1 & & 1 small twin & $1 \mathrm{BCC}$ & & 0 & & \\
\hline Total & & 2 & $2(100)$ & & & & & $1(50)$ & & \\
\hline \multicolumn{11}{|c|}{ Malformations of the genital organs (Q50-Q56) } \\
\hline Q54 & Hypospadias & 2 & & & 2 small twins & & & 2 & & $\begin{array}{l}1 \text { hypospadias repair, cerebral ischemic injury, } \\
\text { severe psychomotor delay; } \\
\text { 1+ AoCoa, hypospadias repair, surgery for CHD, } \\
\text { follow-up. (Co-twin: mild hypospadias, follow-up) }\end{array}$ \\
\hline Total & & 2 & & & & & & $2(100)$ & & \\
\hline \multicolumn{11}{|c|}{ Malformations of the urinary system (Q60-Q64) } \\
\hline Q60.0 & Renal agenesis, unilateral & 2 & 2 & & 2 small twins & & & 2 & & 2 follow-ups \\
\hline Q60.1 & Renal agenesis, bilateral & 3 & 2 & 1 & $\begin{array}{l}1 \text { donor } \\
1 \text { small twin }\end{array}$ & $\begin{array}{l}1 \mathrm{FLS} \text {, misc } \\
1 \mathrm{BCC}\end{array}$ & & 0 & 1 & $1 \mathrm{NND}$ \\
\hline Q60.4 & Renal hypoplasia, bilateral & 1 & 1 & & 1 small twin & & & 1 & & Chronic renal failure, dialysis \\
\hline \multirow[t]{3}{*}{ Q61.4 } & Renal dysplasia & 4 & & & & & & & & \\
\hline & Multicystic bilateral unilateral & & 2 & & 2 small twins & & & 2 & & 2 NND \\
\hline & Multicystic unilateral & & 2 & & $\begin{array}{l}1 \text { donor } \\
1 \text { small twin }\end{array}$ & $1 \mathrm{FLS}$ & & 2 & & 2 follow-ups \\
\hline Q64.10 & Exstrophy of bladder & 2 & 1 & 1 & & $1 \mathrm{BCC}$ & $1 \mathrm{TOP}$ & 0 & 0 & \\
\hline Q64.2 & Congenital posterior urethral valves & 3 & 2 & 1 & 1 large twin & $\begin{array}{l}1 \mathrm{TOP} \\
1 \mathrm{BCC}\end{array}$ & 1 IUFD & 0 & 0 & \\
\hline Total & & 15 & $12(80)$ & $3(20)$ & & & & $7(58)$ & $1(33)$ & \\
\hline
\end{tabular}




\section{TABLE 2}

Continued

\begin{tabular}{|c|c|c|c|c|c|c|c|c|c|c|}
\hline Code & Prenatal findings & Total & $\begin{array}{l}\text { Group } \\
1 N(\%)\end{array}$ & $\begin{array}{l}\text { Group } \\
2 N(\%)\end{array}$ & $\begin{array}{l}\text { Associated } \\
\text { findings }\end{array}$ & $\begin{array}{l}\text { Group } 1 \\
\text { pregnancy } \\
\text { outcome }\end{array}$ & $\begin{array}{l}\text { Group 2 } \\
\text { pregnancy } \\
\text { outcome }\end{array}$ & $\begin{array}{l}\text { Group } \\
1 \text { live } \\
\text { births } \\
\mathrm{N}(\%)\end{array}$ & $\begin{array}{l}\text { Group } \\
2 \text { live } \\
\text { births } \\
N(\%)\end{array}$ & Postnatal outcome \\
\hline \multicolumn{11}{|c|}{ Malformations of the muscoloskeletal system (Q65-Q79) } \\
\hline Q72 & $\begin{array}{l}\text { Reduction defects of lower limb } \\
\text { Tibial agenesis }\end{array}$ & 1 & & 1 & 1 recipient & & $1 \mathrm{BCC}$ & & 0 & (Co-twin NND) \\
\hline Q74.3 & Arthrogryposis multiplex congenital & 2 & 2 & & & $1 \mathrm{BCC}$ & & 1 & & Physiokinesis treatment \\
\hline 076.1 & Klippel Feil syndrome & 2 & 2 & & & $1 \mathrm{BCC}$ & & 1 & & Attention deficit hyperactivity disorder \\
\hline 076.3 & Hemivertebra & 2 & 2 & & & & & 2 & & 2 follow-ups \\
\hline Q79.0 & Congenital diaphragmatic hernia & 1 & & 1 & & & 1 TOP & & 0 & \\
\hline 079.2 & $\begin{array}{l}\text { Omphalocele } \\
\text { Ong }\end{array}$ & 3 & 2 & 1 & & 1 TOP & 1 TOP & 1 & 0 & $\begin{array}{l}\text { + Beckwith-Wiedemann syndrome Omphalocele } \\
\text { repair }\end{array}$ \\
\hline Q79.3 & Gastroschisis & 1 & 1 & & & & & 1 & & NND (Co-twin NND) \\
\hline Q79.59 & $\begin{array}{l}\text { Other congenital malformation of abdominal wall } \\
\text { Limb body wall complex }\end{array}$ & 6 & 3 & 3 & & & $1 \mathrm{TOP}$ & 3 & 2 & 5 NND \\
\hline Total & & 18 & $12(67)$ & 6(33) & & & & $9(75)$ & 2(33) & \\
\hline \multicolumn{11}{|c|}{ Other congenital malformations (O80-O89) } \\
\hline \multirow[t]{3}{*}{ Q87.0 } & $\begin{array}{l}\text { Congenital malformations syndromes } \\
\text { predominantly affecting facial appearance }\end{array}$ & 2 & & & & & & & & \\
\hline & Micrognathia & & 1 & & & $1 \mathrm{BCC}$ & & 0 & & \\
\hline & Micrognathia + low set ears & & 1 & & & 1 IUFD & & & & \\
\hline \multirow[t]{3}{*}{ Q89.9 } & $\begin{array}{l}\text { Congenital malformation, unspecified } \\
\text { Pentalogy of Cantrell }\end{array}$ & 3 & & 1 & & & 1 IUFD & & & \\
\hline & Sacrococcigeal teratoma & & 1 & & & $1 \mathrm{BCC}$ & & 0 & & \\
\hline & Retronucal solid tumor & & & & & & & & & Regression at 1-year, follow-up \\
\hline Total & & 5 & $4(80)$ & 1(25) & & & & $1(25)$ & $0(0)$ & \\
\hline \multicolumn{11}{|c|}{ Chromosomal abnormalities, unclassified elsewhere (Q90-Q99) } \\
\hline Q92.6 & $\begin{array}{l}\text { Marker chromosomes } \\
47, x x+\operatorname{mar}(18 p)\end{array}$ & 1 & 1 & & $\begin{array}{l}1 \text { small twin } \\
\text { malalignment } \\
\text { VSD }\end{array}$ & $1 \mathrm{BCC}$ & & 0 & & \\
\hline Q96 & Turner's syndrome & 5 & 5 & & $\begin{array}{l}5 \text { webbing of } \\
\text { neck }\end{array}$ & $5 \mathrm{BCC}$ & & 0 & & \\
\hline \multicolumn{2}{|l|}{ Total } & 6 & 6 & & & & & $0(0)$ & & \\
\hline
\end{tabular}

Note: Group 1 = number of anomalous twins in MC diamniotic, MC triamniotic, dichorionic triamniotic pregnancies; Group $2=$ number of anomalous twins in MC mono amniotic pregnancies. Small twin/Large twin = anomalous twin of $\mathrm{MC}$ pregnancy with selective intrauterine growth restriction; $\mathrm{D}=$ donor; $\mathrm{R}=$ recipient: anomalous twin of $\mathrm{MC}$ pregnancy with TTTS; NND $=$ neonatal death; TOP $=$ termination of pregnancy; $\mathrm{BCC}=$ bipolar cord coagulation; IUFD = intrauterine fetal death; FLS = fetoscopic laser surgery; VSD = ventricular septal defect; PSARP = posterior sagittal anorectoplasty. Data are expressed as N (number) and percentage in brackets, and refer to the anomalous MC twins. Postnatal outcome of the normal co-twin is reported in brackets.

$\mathrm{Q} 00=1$ pregnancy MC TA; $\mathrm{Q} 22.0=1$ pregnancy MC TA; $\mathrm{Q} 03.0=$ Severe bilateral ventriculomegaly: ventricular size $>15 \mathrm{~mm} ; \mathrm{Q} 21.0=\mathrm{VSD}$, ventricular septal defect 3.0 CCAM = congenital cystic adenomatoid malformation of the lung: $\mathrm{O} 60.1=\mathrm{FLS}$, fetoscopic laser surgery; misc = miscarriage; $\mathrm{Q} 79.2=2$ large omphalocele, 1 small omphalocele. 
listed in Group 2. Table 2 also shows the number of cases ending in IUFD, TOP, or miscarriage, and the number of cases managed with BCC or FLS. The postnatal outcome of the anomalous twins and of the normal co-twins (in brackets) is reported, and the major associated anomalies detected only after birth are also described.

The most frequent anomalies here were those of the nervous system (54 cases, 39\%) and circulatory system (30 cases, $22 \%$ ), followed by the musculoskeletal (13\%) and urinary system (11\%).

The family's choice to opt for BCC was more likely for anomalies of the nervous system (25/54 cases, $46 \%)$, or the circulatory system $(9 / 30,30 \%)$, or chromosomal anomalies $(6 / 6,100 \%)$, all of which were identified on the basis of anomalous ultrasound findings. Of the eight pregnancies with TTTS, four anomalies affected the donor (ICD codes Q04.0, Q22.8, Q60.1, and Q61.4) and four the recipient (codes Q05.2, Q22.0, Q22.5, and Q72). Of the 64 live births with discordant anomalies affecting one system (64/139, 46\%), 20 died immediately after birth or within 2 months, due to the severity of the malformation (codes Q00, Q03.0, Q22.0, Q22.4, Q23.4, and Q79.59). One twin died aged 2 years (code Q01.2). None of the 19 MC MA anomalous twins survived: 14 were lost in utero (eight TOP, four BCC, two IUFD), and five died at birth.

Of the 43 one-system twins surviving infancy, 15 (35\%) underwent surgery. Four children with anomalies of the nervous system (codes Q03.0 and Q03.1) needed educational support at school age. There were two children with heart anomalies (codes Q22.0 and Q22.1) born at 33 and 34 weeks, respectively: one had severe psychomotor delay and the other was affected with spastic bilateral cerebral palsy. Of the two cases with hypospadias (code Q54), one born at 30 weeks (birth weight $1485 \mathrm{~g}$ ) suffered ischemic cerebral injury at birth and has severe psychomotor delay. The other was also diagnosed postnatally with mild aortic coarctation and underwent surgery at 3 months. The child with bilateral renal hypoplasia (code Q60.4) has chronic renal failure and is in dialysis at 6 years of age. The infant with Klippel Feil syndrome (code Q76.1) suffers from hyperactive attention deficit disorder, while the twin born with small omphalocele (code Q79.2) was found to have BeckwithWiedemann syndrome and is in specific follow-up. In this cohort of cases of congenital anomaly affecting one system only, severe neurodevelopmental disability was detected in 3 out of 43 surviving twins (7\%).

Table 3 describes the 16 cases $(16 / 155,10 \%)$ of major discordant structural anomalies affecting multiple systems, occurring in $15 \mathrm{MC}$ DA and in 1 DC TA pregnancy. All 16 had normal conventional karyotyping/aCGH, performed prenatally in 13 cases and postnatally in 3 cases. Of the eight live births (50\%), one died in the neonatal period. The seven surviving twins had surgery or multiple surgeries. One of the seven (14\%) who was delivered at 30 weeks suffers severe psychomotor delay.
Regarding the outcome of the normal MC co-twins, the overall survival rate was $71 \%(111 / 157)$, with generally satisfactory follow-up. The five neonatal deaths occurred in twins delivered before 27 weeks. Of the seven independent twins delivered, two died because of necrotizing enterocolitis in course of sepsis, while the other five are doing well.

\section{Discussion}

This retrospective study was designed to provide a detailed description of the spectrum of major discordant structural anomalies observed prenatally in a large cohort of MC twins, including the management of these pregnancies and the outcome of the twins. Any analysis of the genetic mechanisms allegedly responsible for these discordances was beyond the scope of the study, as was any estimate of prevalence, since the entrance requirement for the study was the discordant anomaly.

Careful and accurate identification and classification of congenital anomalies is crucial, not only to establish the prevalence of malformations, but also to minimize the subjectivity of reports, make inter-center comparisons possible, and simplify data analysis. However, classifying the entire range of congenital anomalies represents an enormous challenge that only increases in the presence of rare and complex malformations or anomalies involving multiple systems. For the purposes of this study, we decided to adopt the ICD-10 system, currently used worldwide for postnatal disease classification, despite its reported limitations that is, the lack of accuracy for coding certain anomalies (mainly those of the circulatory and nervous systems), and lack of inclusion of all malformations (Metcalfe et al., 2014). Despite these limitations, the code sets did enable us to bring together a vast spectrum of different malformations observed prenatally and made interpreting our data much easier.

Despite its inherent limitations with our center being a third-level facility, this study shows that the most common severe anomalies involve the nervous, circulatory, musculoskeletal, and multiple systems. Other studies have described discordant anomalies in twins, but they were not subdivided for chorionicity (Fernandes et al., 2016; Harper et al., 2012). The few articles that do divide for chorionicity describe fewer cases of discordant anomaly than we do here (Gul et al., 2005; Linskens et al., 2011). One registerbased study (Glinianaia et al., 2008) and a recent report on MC twins with suspected discordant malformations (which makes no distinction between major abnormalities and minor ultrasound findings such as single umbilical artery, choroid plexus cyst, double renal pelvis, and amniotic band syndrome) confirmed that nervous system, circulatory system, and multiple anomalies are much more common (Peng et al., 2016). In our series, only eight cases of major anomalies (5\%) were complicated with TTTS (four affected the donor and four the recipient), since we excluded cases of 
TABLE 3

Major Discordant Structural Anomalies Affecting Multiple Systems (N 16)

\begin{tabular}{|c|c|c|c|c|c|c|}
\hline & Prenatal findings & $\mathrm{N}$ & $\begin{array}{l}\text { Associated } \\
\text { findings }\end{array}$ & $\begin{array}{l}\text { Pregnancy } \\
\text { outcome }\end{array}$ & $\begin{array}{l}\text { Live births } \\
N(\%)\end{array}$ & Postnatal outcome \\
\hline \multirow[t]{5}{*}{$\begin{array}{l}\text { Circulatory system } \\
+ \text { nervous } \\
\text { system }\end{array}$} & $\begin{array}{l}\text { Truncus arteriosus }+ \\
\text { amartomatous dysplasia of } \\
\text { left hemisphere }\end{array}$ & 1 & Small twin & $\mathrm{BCC}$ & 0 & - \\
\hline & $\begin{array}{l}\text { Tetralogy of Fallot }+ \text { aracnoid } \\
\text { cyst }\end{array}$ & 1 & - & & 1 & Surgery for CHD, follow-up \\
\hline & $\begin{array}{l}\text { Interruption of aortic arch + } \\
\text { hypoplastic CC }\end{array}$ & 1 & - & $\mathrm{BCC}$ & 0 & - \\
\hline & $\begin{array}{l}\text { Malaligment VSD + vermian } \\
\text { hypoplasia }\end{array}$ & 2 & Small twins & $1 \mathrm{BCC}$ & 1 & Surgery for CHD, follow-up \\
\hline & $\begin{array}{l}\text { Single ventricle + vermian } \\
\text { hypoplasia }\end{array}$ & 1 & - & $\mathrm{BCC}$ & 0 & - \\
\hline \multirow{4}{*}{$\begin{array}{l}\text { Circulatory system } \\
+ \text { digestive } \\
\text { system }\end{array}$} & $\begin{array}{l}\text { Aortic coarctation }+ \\
\text { esophageal atresia }\end{array}$ & 1 & - & & 1 & $\begin{array}{l}\text { Esophageal atresia repair, } \\
\text { surgery for CHD, follow-up }\end{array}$ \\
\hline & $\begin{array}{l}\text { Tetralogy of Fallot }+ \\
\text { esophageal atresia }\end{array}$ & 1 & Small twin & & 1 & $\begin{array}{l}\text { Esophageal atresia repair, } \\
\text { surgery for CHD, moderate } \\
\text { intellectual disability }\end{array}$ \\
\hline & $\begin{array}{l}\text { Single ventricle + esophageal } \\
\text { atresia }\end{array}$ & 1 & - & & 1 & NND \\
\hline & DORV + duodenal obstruction & 1 & Small twin & & 1 & $\begin{array}{l}\text { Duodenal anastomosis, } \\
\text { surgery for } \mathrm{CHD} \text {, follow-up }\end{array}$ \\
\hline \multirow{2}{*}{$\begin{array}{l}\text { Circulatory system } \\
+ \text { other } \\
\text { congenital } \\
\text { anomalies }\end{array}$} & $\begin{array}{l}\text { Pulmonary atresia intact } \\
\text { ventricular septum }+ \\
\text { micrognathia }\end{array}$ & 1 & - & IUFD & 0 & - \\
\hline & $\begin{array}{l}\text { Truncus arteriosus }+ \\
\text { monolateral MCK + left leg } \\
\text { agenesis, left arm } \\
\text { hypoplasia }\end{array}$ & 1 & - & IUFD & 0 & - \\
\hline \multirow[t]{2}{*}{$\begin{array}{l}\text { Digestive system }+ \\
\text { other congenital } \\
\text { anomalies }\end{array}$} & $\begin{array}{l}\text { Esophageal atresia + severe } \\
\text { monolateral hydronephrosis }\end{array}$ & 1 & - & & 1 & $\begin{array}{l}\text { Plus imperforate anus, } \\
\text { esophageal atresia repair, } \\
\text { PSARP nephrectomy at } 4 \\
\text { months follow-up }\end{array}$ \\
\hline & $\begin{array}{l}\text { Esophageal atresia + short } \\
\text { left forearm, clenched } \\
\text { hands and feet }\end{array}$ & 1 & Small twin & & 1 & $\begin{array}{l}\text { Esophageal atresia repair, } \\
\text { severe psychomotor delay } \\
\text { (Co-twin: mild psychomotor } \\
\text { delay) }\end{array}$ \\
\hline \multirow[t]{2}{*}{ Other } & $\begin{array}{l}\text { Complex scoliosis }+ \\
\text { mono-lateral renal agenesis }\end{array}$ & 1 & - & $\mathrm{BCC}$ & 0 & - \\
\hline & $\begin{array}{l}\text { Severe ventriculomegaly }+ \\
\text { subcutaneous edema }\end{array}$ & 1 & - & TOP & 0 & - \\
\hline Total & & 16 & & & $8(50)$ & \\
\hline
\end{tabular}

Note: VSD = ventricular septal defect, $C C=$ corpus callosum, $C H D=$ congenital heart disease, MCK = multicystic kidney, OE = esophageal atresia, IUFD = intrauterine fetal death, BCC = bipolar cord coagulation, TOP = termination of pregnancy, PSARP = posterior sagittal anorectoplasty. Small twin $=$ anomalous twin of monochorionic pregnancy with selective intrauterine growth restriction. Data are expressed as number $(N)$ and percentage in brackets and refer to the anomalous twin. Postnatal outcome of the normal co-twin is reported in brackets.

recipient-twin cardiomyopathy from the study. Interestingly, in the retrospective cohort reported of TTTS pregnancies treated with laser surgery (Patel et al., 2012), the prevalence of non-cardiac anomalies (15 major, 4 minor) in the 377 live births (5\%) was higher in donors than in recipients ( $8.5 \%$ vs. $2.0 \%)$. In the series described by Kontopoulos et al. (2008), the incidence of Dandy-Walker syndrome was 200 times higher in complicated MC twins than in singletons, and the affected twin was the smaller (donor or IUGR) in $8 / 10$ cases. In our cohort, too, of the 41 pregnancies with associated sIUGR, the small twin was affected by a major anomaly in $36 / 41$ cases (88\%). This association of congenital structural malformations with intrauterine growth restriction (and the hypoxic environment arising from placental dysfunction) on the one hand, and with TTTS and impaired perfusion in the donor twin on the other, points to a role for hypoxia and/or uneven perfusion in determining congenital anomalies. Although experimental models are non-transferable to humans, studies with animals have shown that hypoxia during pregnancy can induce congenital malformations. The most commonly reported anomalies are limb reduction defects, cleft lip/palate, heart defects, death of neuronal population and cerebral anomalies, and hemorrhage in the external genitalia with subsequent hypospadias (Webster \& Abela, 2007).

Since only $6 \%$ of women in our series underwent IVF, this small number of cases makes it impossible to assess a possible statistical correlation between IVF and specific anomalies.

A discordant abnormal karyotype in association with major structural anomalies was found in 6 of the 94 twins (6\%) undergoing invasive prenatal diagnosis. In five of these 
six cases, the anomaly was Turner's syndrome, confirming previous observations indicating that monosomy $\mathrm{X}$ is the most frequent heterokaryotypia in MZ twins (Gringas \& Chen, 2001). With all the limits arising from a population examined after 14 weeks' gestation, which makes the loss of affected fetuses likely before referral, we found no cases of trisomy 21 or of discordant copy number variations that might have caused the phenotypic discordance, which is an interesting but still unresolved issue (Shi et al., 2017; Veenma et al., 2012).

Family choices and pregnancy outcomes were strongly influenced by the kind of anomaly affecting the twin and the type of MC pregnancy (whether monoamnotic or diamniotic). More than one-third of women requested selective feticide, either to avoid the potential risk of in utero fetal death following conservative management (and the risk of demise or severe morbidity in the co-twin), or to avoid the risk of a live-birth twin with major defects. Eight of the 16 terminated pregnancies were monoamniotic, a condition that presents more technical difficulties and additional risks than a diamniotic pregnancy when performing selective feticide (Valsky et al., 2011). Furthemore, the combination of a major structural anomaly (even when nonlethal and potentially correctable postnatally) with selective IUGR was perceived by some parents as being too much to bear, and swayed parental decision making in the favor of selective feticide or termination. Parents' concerns about the anomalous twin, together with the inherent possible complications of MC pregnancies, such as spontaneous double or single fetal loss, or co-twin demise after cord occlusion, resulted in less than $50 \%$ of anomalous fetuses being born alive. All these aspects, together with the rarity of the discordant anomaly in MC twins, limit clinicians' ability to inform parents of the potential course and outcome in a particular malformation. This limitation could probably be overcome by combining the data from large centers to compile larger cases series for specific anomaly.

In total, 12 out of 22 neonatal deaths (54\%) were caused by lethal anomalies in conservative management, while the remainder was due to prematurity. Overall, of the 50 surviving infants, 22 (44\%) underwent major surgery or multiple surgeries. Four survivors ( $8 \%$, three of whom had surgery) now suffer from severe neurologic morbidity. The vast majority of normal co-twin survivors had a good outcome and are free from neurologic morbidity.

In conclusion, this study shows that a wide spectrum of major discordant structural anomalies can occur in MC pregnancies, and operators involved in the care of these pregnancies should be aware of this. Parents are faced with complex decisions regarding the fate of the anomalous twin and the course of the pregnancy. While around half of the survivors with structural anomalies did not undergo surgery or suffer severe neurologic morbidity, many of these infants are still in follow-up and will need long-term care from their families and from health services.

\section{References}

Alexander, J. M., Ramus, R., Susan, M., Cox, S. M., \& Gilstrap, L. C. (1997). Outcome of twin gestations with a single anomalous fetus. American Journal of Obstetrics and Gynecology, 177, 849-852.

Castillo-Fernandez, J. E., Spector, T. D., \& Bell, J. T. (2014). Epigenetics of discordant monozygotic twins: Implications for disease. Genome Medicine, 6, 2-16.

Conte, G., Parazzini, C., Falanga, G., Cesaretti, C., Izzo, G., Rustico, M. A., ... Righini, A. (2016). Diagnostic value of prenatal MR imaging in the detection of brain malformations in fetuses before the 26th week of gestational age. American Journal of Neuroradiology, 37, 946-951.

Fernandes, T. R., Carvalho, P. R., Flosi, F. B., \& Baiao, A. E. (2016). Perinatal outcome of discordant anomalous twins: A single-center experience in developing country. Twin Research and Human Genetics, 19, 389-392.

Gentilin, B., Guerneri, S., Bianchi, V., Natacci, F., Colombo, A., Fogliani, R., ... Lalatta, F. (2008). Discordant phenotype and karyotype of monozygotic twins characterized by the unequal distribution of two cell lines investigated by different methods: A review. Twin Research and Human Genetics, 11, 352-356.

Glinianaia, S. V., Rankin, J., \& Wright, C. (2008). Congenital anomalies in twins: A register-based study. Human Reproduction, 23, 1306-1311.

Gratacos, E., Ortiz, J. U., \& Martinez, J. M. (2012). A systematic approach to the differential diagnosis and management of the complications of monochorionic twin pregnancies. Fetal Diagnosis and Therapy, 32, 145-155.

Gringras, P., \& Chen, W. (2001). Mechanism for differences in monozygous twins. Early Human Development, 64, 105117.

Gul, A., Cebeci, A., Aslan, A., Polat, I., Sozen, I., \& Ceylan, Y. (2005). Perinatal outcomes of twin pregnancies discordant for major anomalies. Fetal Diagnosis and Therapy, 20, 244248.

Hall, J. G. (2003). Twinning. Lancet, 362, 735-743.

Harper, L. M., Odibo, A. O., Roehl, K. A., Longman, R. E., Macones, G. A., \& Cahill, A. G. (2012). Risk of preterm delivery and growth restriction in twins discordant for structural anomalies. American Journal of Obstetrics and Gynecology, 206, 70.e1-70.e5.

Hilmann, S. C., McMullan, D. J., Hall, G., Togneri, F. S., James, N., Maher, E. J., \& Kilby, M. D. (2013). Use of prenatal chromosomal microarray: Prospective cohort study and systematic review and meta-analysis. Ultrasound in Obstetrics and Gynecology, 41, 610-620.

Kantopoulos, E. V., Quintero, R. A., Salihu, H. M., Bornick, P. W., \& Allen, M. H. (2008). Dandy-Walker syndrome and monochorionic twins: Insights into a possible etiological mechanism. Journal of Maternal-Fetal \& Neonatal Medicine, 21, 839-842.

Khalil, A., Rodgers, M., Baschat, A., Bhide, A., Gratacos, E., Hecher, K. ... Ville, Y. (2016). ISUOG practice guidelines: Role of ultrasound in twin pregnancy. Ultrasound in Obstetrics and Gynecology, 47, 247-263. 
Lanna, M. M., Rustico, M. A., Dell'Avanzo, M., Schena, V., Faiola, S., Consonni, D., ... Ferrazzi, E. M. (2012). Bipolar cord coagulation for selective feticide in complicated monochorionic twin pregnancies: 118 consecutive cases at a single center. Ultrasound in Obstetrics and Gynecology, 39, 407-413.

Linskens, I. H., Elburg, R. M., Oepkes, D., Vugt, G. M., \& Haak, M. C. (2011). Expectant management in twin pregnancies with discordant structural fetal anomalies. Twin Research and Human Genetics, 14, 283-289.

Machin, G. (2009). Non-identical monozygotic twins, intermediate twin pairs, zygosity testing, and the non-random nature of monozygotic twinning: A review. American Journal of Medical Genetics Part C, Seminars in Medical Genetics, 151, 110-127.

Manning, N., \& Archer, N. (2016). Cardiac manifestations of twin-to-twin transfusion syndrome. Twin Research and $\mathrm{Hu}$ man Genetics, 19, 246-254.

Marlow, N. (2013). Measuring neurodevelopmental outcome in neonatal trials: A continuing and increasing challenge. Archives of Disease in Childhood: Fetal and Neonatal Edition, 98, 554-558.

Metcalfe, A., Sibbald, B., Lowry, R. B., Tough, S., \& Bernier, F. P. (2014). Validation of congenital anomaly coding in Canada's administrative database compared with a congenital anomaly registry. Birth Defects Research Part A: Clinical and Molecular Teratology, 100, 59-66.

Patel, S., Randolph, L. M., Benirschke, K., Llanes, A., Yedigarova, L., \& Chmait, R. H. (2012). Prevalence of noncardiac structural anomalies in twin-twin transfusion syndrome. Journal of Ultrasound in Medicine, 31, 555-560.

Peng, R., Zhou, Y., Xie, H., Zheng, J., Xie, Y. J., \& Yang, J. B. (2016). MCDA twins with discordant malformations: Submicroscopic chromosomal anomalies detected by chromosomal microarray analysis and clinical outcomes. Prenatal Diagnosis, 36, 766-774

Pharoah, P. O., \& Dundar, Y. (2009). Monozygotic twinning, cerebral palsy and congenital anomalies. Human Reproduction Update, 15, 639-648.

Rustico, M. A., Consonni, D., Lanna, M. M., Faiola, S., Schena, V., Scelsa, B., ... Ferrazzi, E. (2017). Selective intrauterine growth restriction in monochorionic twins: Changing patterns in umbilical artery Doppler flow and outcomes. $\mathrm{Ul}$ trasound in Obstetrics and Gynecology, 5, 1352-1357.

Rustico, M. A., Lanna, M. M., Faiola, S., Schena, V., Dell'Avanzo, M., Mantegazza, V., ... Ferrazzi, E. (2012). Fetal and maternal complications after selective fetoscopic laser surgery for twin-to-twin transfusion syndrome: A single-center experience. Fetal Diagnosis and Therapy, 31, 170-178.
Shi, X., Li, L., Huang, X., Chen, B., Zhou, Y., \& Fang, Q. (2017). Fetal aneuploidy: A comparison of dichorionic twins and monochorionic twins. Fetal Diagnosis and Therapy, 44, 124-128.

Silva, S., Martins, Y., Matias, A., \& Blickstein, I. (2011). Why are monozygotic twins different? Journal of Perinatal Medicine, 39, 195-202.

Slaghekke, F., Lopriore, E., Lewi, L., Middeldorp, J. M., van Zwet, E. W., Weingertner, A. S., ... Oepkes, D. (2014). Fetoscopic laser coagulation of the vascular equator versus selective coagulation for twin-to-twin transfusion syndrome: An open-label randomized controlled trial. Lancet, 383, 2144-2151.

Sperling, L., Kiil, C., Larsen, L. U., Brocks, V., Wojdemann, K. R., Qvist, I., ... Tabor, A. (2007). Detection of chromosomal abnormalities, congenital abnormalities and transfusion syndrome in twins. Ultrasound in Obstetrics and Gynecology, 29, 517-526.

Springer, S., Mlczoch, E., Krampl-Bettelheim, E., MailathPokorny, M., Ulm, B., Worda, C., ... Worda, K. (2014). Congenital heart disease in monochorionic twins with and without twin-to-twin transfusion syndrome. Prenatal Diagnosis, 34, 994-999.

Valsky, D. V., Martinez-Serrano, M. J., Sanz, M., Eixarch, E., Acosta, E. R., Martinez, J. M., ... Gratacos, E. (2011). Cord occlusion followed by laser cord transection in monochorionic monoamniotic discordant twins. Ultrasound in Obstetrics and Gynecology, 37, 684-688.

Veenma, D., Brosens, E., de Jong, E., van de Ven, C., Meeussen, C., Cohen-Overbeek, T., ... de Klein, A. (2012). Copy number detection in discordant monozygotic twins of congenital diaphragmatic hernia (CHD) and esophageal atresia (EA) cohorts. European Journal of Human Genetics, 20, 298-304.

Webster, W. S., \& Abela, D. (2007). The effect of hypoxia in development. Birth Defects Research Part C: Embryo Today, $81,215-228$.

Wood, S., Tang, S., Ross, S., \& Sauve, R. (2014). Stillbirth in twins, exploring the optimal gestational age for delivery: A retrospective cohort study. BJOG, 121, 1284-1295.

World Health Organization. (2016). International Statistical Classification System of Diseases and Related Health Problems, tenth revision (ICD-10). Geneva: Author.

Zwijnenburg, P. J., Meijers-Heijboer, H., \& Boomsma, D. I. (2010). Identical but not the same: The value of discordant monozygotic twins in genetic research. American Journal of Medical Genetics Part B, Neuropsychiatric Genetics, 153, 1134-1149. 